\title{
An Estimate from Above of the Number of Periodic Orbits for Semi-Dispersed Billiards
}

\author{
Luchezar Stojanov \\ Institute of Mathematics, Bulgarian Academy of Sciences, BG-1090 Sofia, Bulgaria
}

Abstract. For a large class of semi-dispersed billiards an exponential estimate from above is found for the number of periodic points of the billiard ball map.

\section{Introduction and Main Results}

Let $Q$ be a domain (bounded or unbounded) in $\mathbb{R}^{d}, d \geqq 2$, with the boundary

$$
\partial Q=\Gamma=\Gamma_{1} \cup \Gamma_{2} \cup \cdots \cup \Gamma_{s} \quad(s \geqq 3),
$$

where each $\Gamma_{i}$ is a compact convex $C^{2}$-smooth $(d-1)$-dimensional submanifold of $\mathbb{R}^{d}$ with piecewise smooth boundary $\partial \Gamma_{i}$, and

$$
\Gamma_{i} \cap \Gamma_{j} \subset \partial \Gamma_{i} \cup \partial \Gamma_{j}
$$

whenever $i \neq j$. Each $\partial \Gamma_{i}$ is the union of a finite number of compact $(d-2)$ dimensional submanifolds of $\mathbb{R}^{d}$. If $\partial \Gamma_{i} \neq \varnothing$, then clearly $\Gamma_{i}$ is the boundary of a compact convex domain in $\mathbb{R}^{d}$.

Main Assumption. In the sequel we assume that each $\Gamma_{i}$ is contained in the boundary of a convex domain in $\mathbb{R}^{d}$. Therefore if $K_{i}$ is the convex hull of $\Gamma_{i}$, then $\Gamma_{i} \subset \partial K_{i}$.

The points of

$$
\stackrel{\circ}{\Gamma}=\left(\Gamma_{1} \backslash \partial \Gamma_{1}\right) \cup \cdots \cup\left(\Gamma_{s} \backslash \partial \Gamma_{s}\right)
$$

will be called regular points of $\Gamma$. For $q \in \Gamma^{\circ}$ we denote by $N(q)$ the normal unit vector to $\Gamma$ at $q$ directed to the interior of $Q$. With respect to this framing the second fundamental form of $\Gamma$ is non-negative definite at each $q \in \dot{\Gamma}$.

We consider the billiard in $Q$, that is the dynamical system generated by the motion of material point in $Q$ (see $[4,13]$ ). The point is moving with constant velocity in the interior of $Q$ with reflections at $\partial Q$ according to the rule "the angle of incidence is equal to the angle of reflection."

\footnotetext{
* Partially supported by the Bulgarian Commitee of Sciences, Grant No. 52
} 


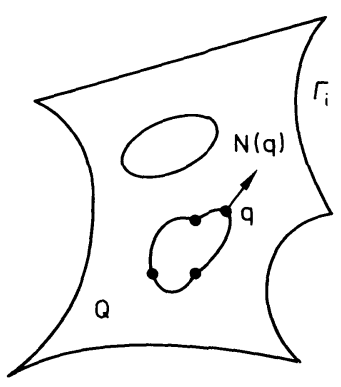

a

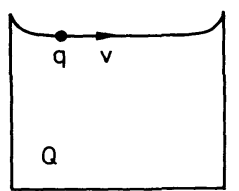

a

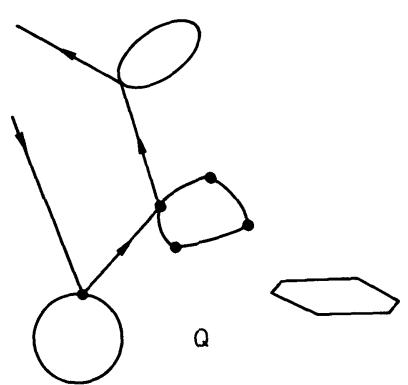

b

Fig. 1

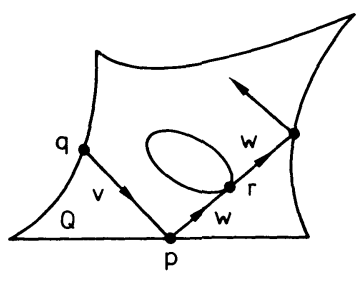

b

Fig. 2

Denote by $\langle.,$.$\rangle the scalar product in \mathbb{R}^{d}$ and by $L_{q} \Gamma$ the tangent hyperplane to $\Gamma$ at $q$. Then $L_{q} \Gamma=q+L_{q}^{\prime} \Gamma$, where $L_{q}^{\prime} \Gamma$ is a linear subspace of $\mathbb{R}^{d}$, and $T_{q} \Gamma=\{q\} \times L_{q}^{\prime} \Gamma$ is the tangent space to $\Gamma$ at $q$.

A point $x=(q, v) \in \Gamma \times S^{d-1}$ will be called admissible if it satisfies the following two conditions:

(i) $q$ is regular and $\langle N(q), v\rangle \geqq 0$;

(ii) if $\langle N(q), v\rangle=0$, then there exists in $\Gamma$ a neighbourhood $U$ of $q$ such that $U \cap L_{q} \Gamma=\{q\}$.

Set

$$
M^{\prime}=\left\{(q, v) \in \stackrel{\circ}{\Gamma} \times S^{d-1}:\langle N(q), v\rangle \geqq 0\right\} .
$$

Denote by $M$ the set of $x=(q, v) \in M^{\prime}$ such that if $\gamma(x)$ is the billiard semi-trajectory in $Q$ starting at $q$ in the direction $v$, then $\gamma(x) \cap \Gamma \subset \stackrel{\circ}{\Gamma}, \gamma(x)$ intersects $\Gamma$, and whenever $\gamma(x)$ is passing through a point $p \in \Gamma$ with reflected direction $w$, then $(p, w)$ is an admissible point of $\Gamma \times S^{d-1}$. For $x \in M$ let $p$ be the first point of reflection of $\gamma(x)$, that is $p \in \gamma(x) \cap \stackrel{\circ}{\Gamma}$ and the open segment $(q, p)$ is contained in the interior of $Q$. Set

$$
T(x)=T(q, v)=(p, w),
$$

where $w=v-2\langle N(p), v\rangle N(p)$. Thus we obtain a map

$$
T: M \rightarrow M^{\prime}
$$

which is called the billiard ball map related to $Q$. In fact, it is more natural to 
consider $T$ as a map

$$
T: M_{0} \rightarrow M_{0}
$$

where $M_{0}=\bigcap_{m=0}^{\infty} T^{-m}(M)$. Note that if $Q$ is bounded, then $M^{\prime} \backslash M$ has a Lebesgue measure zero (cf. [4]).

If $Q$ is a bounded and $\Gamma$ is strictly convex (convex) at each $q \in \Gamma_{\Gamma}^{\circ}$, then the billiard in $Q$ is called dispersed (respectively semi-dispersed). Dispersed billiards were introduced by Sinai [15]. Various properties of dispersed and semi-dispersed billiards were studied by many authors in connection with some problems in statistical mechanics and mathematical physics (cf. $[4,2,3,5,6,9-18]$ and the references given there).

For each integer $k \geqq 2$ denote by $\mathscr{A}_{k}$ the set of those $k$-tuples $\alpha=\left(i_{1}, \ldots i_{k}\right)$ such that $i_{j}=1,2, \ldots, s$ for all $j, i_{j} \neq i_{j+1}$ for $j=1, \ldots, k-1$ and $i_{k} \neq i_{1}$. Let

$$
\pi: \Gamma \times S^{d-1} \rightarrow \Gamma
$$

be the natural projection. A point $x=(q, v) \in M_{0}$ is called a periodic point of type $\alpha$ for $T$ if $T^{k}(x)=x$ and

$$
q_{j}=\pi \circ T^{j-1}(x) \in \Gamma_{i_{j}}
$$

for any $j=1,2, \ldots, k$. If the segment $\left[q_{j}, q_{j+1}\right]$ is tangent to $\Gamma$ at $q_{j}$, then $q_{j}$ will be called a tangent reflection point of $\gamma(x)$, otherwise it will be called a proper reflection point of $\gamma(x)$.

The main result in this paper is the following

Theorem 1.1. Let $Q$ satisfy the above assumptions and let $\alpha \in \mathscr{A}_{k}$. Let there exist two different periodic points $(q, v)$ and $(p, w)$ of type $\alpha$ for $T$ and let $q_{j}=\pi \circ T^{j-1}(q, v)$, $p_{j}=\pi \circ T^{j-1}(p, w), j=1,2, \ldots$ Then $v=w$, and for every $j \geqq 1$ the segments $\left[q_{j}, q_{j+1}\right]$ and $\left[p_{j}, p_{j+1}\right]$ are parallel. If $q_{j}$ is a proper reflection point, then $t q_{j}+(1-t) p_{j} \in \Gamma_{i_{j}}$ for all $t \in(0,1)$ sufficiently close to 1 . If all $q_{j}$ are proper reflection points, then for every $t \in(0,1)$ sufficiently close to 1 the points $(t q+(1-t) p, v)$ are periodic points of type $\alpha$ for $T$ generating periodic billiard trajectories in $Q$ of the same length, and these trajectories have parallel corresponding segments.

In other words, for every $\alpha \in \mathscr{A}_{k}$ there are three possibilities: (a) there are no periodic points of type $\alpha$; (b) there exists exactly one periodic point of type $\alpha$; (c) the periodic points of type $\alpha$ generate a family (which might be discrete, see Fig. 3 (a)) of parallel periodic billiard trajectories in $Q$ of the same period (length). The assumption that $q_{j}$ is a proper reflection point is essential for the second part of the theorem (cf. again Fig. 3 (a)).

Since every periodic billiard trajectory has at least two proper reflection points, the following is an immediate consequence of the above theorem.

Corollary 1.2. If $\alpha=\left(i_{1}, \ldots, i_{k}\right) \in \mathscr{A}_{k}$ and $\Gamma_{i_{j}}$ is strictly convex for some $j=1, \ldots, k$, then there exists at most one periodic point of type $\alpha$ for $T$.

We should mention that Theorem 1.1 and Corollary 1.2 fail if we drop our main assumption (cf. Fig. 3 (b)). They fail also if one considers domains $Q$ in an 


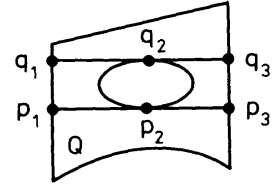

a

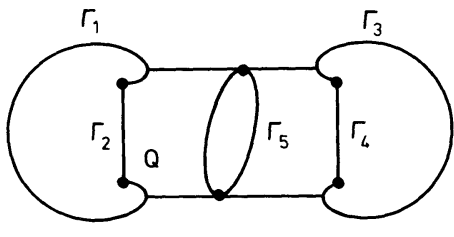

b

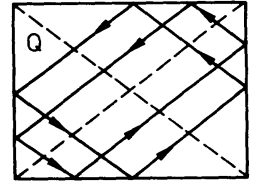

C

Fig. 3

arbitrary Riemannian manifold. It is easy to construct counterexamples with $Q \subset$ Tor $^{2}$ or $Q \subset S^{2}$.

If $(q, v)$ and $(p, w)$ are periodic points of period $k$ for $T$, we will say that $(q, v)$ and $(p, w)$ are equivalent if they are of the same type and generate parallel periodic billiard trajectories of equal lengths. Denote by $P_{k}=P_{k}(Q)$ the number of equivalent classes of periodic points of period $k$ for $T$.

Counting the cardinality of $\mathscr{A}_{k}$ and applying Theorem 1.1 one gets immediately the following.

Corollary 1.3. Let $Q$ satisfy the assumptions at the beginning of this section. Then for every integer $k \geqq 3$ we have

$$
P_{k} \leqq s(s-1)^{k-2}(s-2)<(s-1)^{k} .
$$

In particular, $\lim \sup \left(\log P_{k} / k\right) \leqq s-1$.

There is a large class of unbounded domains $Q$ for which $P_{k}=s(s-1)^{k-2}(s-2)$ for all $k \geqq 3$. One may take for example all domains $Q$ which are exteriors of several disjoint strictly convex compact domains in $\mathbb{R}^{d}$ and satisfy the condition $(H)$ below (cf. [5]). Note that if $\Gamma_{i}$ is strictly convex for every $i$, then $P_{k}$ is exactly the number of all periodic points of period $k$ for $T$.

The growth rate of the number $P(t)$ of closed geodesics of length $\leqq t$ on Riemannian manifolds, as well as that of the number $P_{k}(f)$ of periodic points of period $k$ for diffeomorphisms $f$ on compact manifolds, have been studied by many authors and in different contexts (cf. Katok [7, 8] for more details and some historical remarks). For example, for manifolds of negative curvature $\lim _{t \rightarrow \infty} P(t) / t$ exists and equals the topological entropy of the geodesic flow (Margulis [12]). If $f$ is an Axiom $A$ diffeomorphisms, then $\lim \sup \left(\log P_{k}(f) / k\right)$ equals the topological entropy $h(f)$ of $f$ (Bowen [1]). Katok [7] proved that if $f$ is a $C^{1+\varepsilon}(\varepsilon>0)$ diffeomorphism of a compact manifold and $\mu$ is a Borel probability $f$-invariant measure with non-zero Lyapunov exponents, then $\lim \sup \left(\log P_{k}(f) / k\right.$ is not less than the metric entropy $h_{\mu}(f)$. Concerning the billiard ball map $T$ we do not know any estimates of $P_{k}(T)$ by means of the (metric) entropy of $T$.

As N. Chernov pointed out, Theorem 1.1 has some consequences in the case when $\Gamma_{i}$ are cylinders, which may have some applications to the study of systems of elastic hard spheres (cf. $[17,11]$ ).

Let $Q \subset \mathbb{R}^{2}$ and $\partial Q=\Gamma_{1} \cup \cdots \cup \Gamma_{s}$. Every $\Gamma_{i}$ is a smooth curve in $\mathbb{R}^{2}$ which may have one or two endpoints. If $i \neq j$ and $\Gamma_{i} \cap \Gamma_{j} \neq \varnothing$, then $\Gamma_{i} \cap \Gamma_{j}$ consists of 


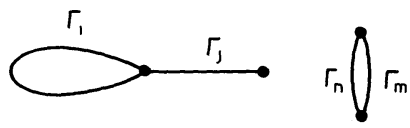

a

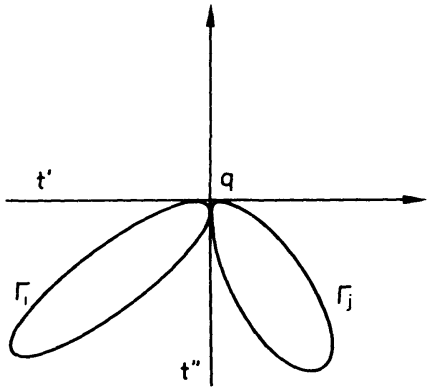

b

Fig. 4

one or two points. Let $q \in \Gamma_{i} \cap \Gamma_{j}$. We will say that the pair $\left(\Gamma_{i}, \Gamma_{j}\right)$ is singular at $q$ if there is a common tangent line $t$ to $\Gamma_{i}$ and $\Gamma_{j}$ at $q$ such that $\Gamma_{i}$ and $\Gamma_{j}$ lie in different halfplanes with respect to $t$. Note that there could be two different common tangents to $\Gamma_{i}$ and $\Gamma_{j}$ at $q$ (cf. Fig. 4).

Corollary 1.4. Let $Q$ be bounded, $Q \subset \mathbb{R}^{2}$, and let $\Gamma_{i}$ be strictly convex for every $i=1, \ldots, s$. Suppose moreover that for all $i \neq j$ with $\Gamma_{i} \cap \Gamma_{j} \neq \varnothing$ the pair $\left(\Gamma_{i}, \Gamma_{j}\right)$ is non-singular at any point $q \in \Gamma_{i} \cap \Gamma_{j}$. Then there exists constants $c>0, b>0$ such that

$$
\tilde{P}_{t} \leqq(s-1)^{c t+b} \quad(t>0),
$$

where $\tilde{P}_{t}$ denotes the number of those $(q, v) \in M_{0}$ which generate periodic billiard trajectories in $Q$ with lengths $\leqq t$.

An exponential estimate from below of $P_{k}$ for semi-dispersed billiards in $\mathbb{R}^{2}$ is found by Bunimovich et al. [3]. It is also shown in [3] that the periodic points of the billiard map $T$ are dense in the phase space $M_{0}$. These results are obtained as consequences of the existence of Markov partitions for such billiards established in [3].

Note that Theorem 1.1 works also in the case when $Q$ is a polyhedron in $\mathbb{R}^{d}$, however in this case much better estimates for $P_{k}$ and $\widetilde{P}_{t}$ were found by Katok [9].

Finally, consider the case when $Q=\mathbb{R}^{d} \bigcup_{i=1}^{s} K_{i}$, where $K_{i}$ are disjoint strictly convex compact domains in $\mathbb{R}^{d}$ with $C^{2}$-smooth boundaries $\partial K_{i}=\Gamma_{i}$. In this case Ikawa [5] proved Theorem 1.1 under the following additional assumption:

$$
\text { (H) }\left\{\begin{array}{l}
\text { For } i, j \in\{1, \ldots, s\}, i \neq j, \text { the convex hull of } \\
K_{i} \cup K_{j} \text { contains no points of the set } \\
\cup\left\{K_{m}: m \neq i, j\right\} .
\end{array}\right.
$$

Using this fact and the technique of [5], Ikawa [6] proved that in the latter case there exists $\varepsilon>0$ such that the domain $\{z \in \mathbb{C}: 0<\operatorname{Im} z<\varepsilon\}$ contains infinitely many poles of the scattering matrix $S(z)$ related to the wave equation in $Q$ with Neumann boundary conditions on $\partial Q$. On the other hand, it follows by [14] that for generic $Q$ in $\mathbb{R}^{d}$ (see [14] for the precise definition of "generic") all periodic billiard trajectories in $Q$ have only proper reflection points. It seems that using this fact, Theorem 1.1 and the technique of Ikawa $[5,6]$ one can derive that for generic $Q$ 
in $\mathbb{R}^{d}$ (but without assuming $\left.(H)\right)$ there always exists $\varepsilon>0$ such that the scattering matrix $S(z)$ related to $Q$ has infinitely many poles $z$ with $0<\operatorname{Im} z<\varepsilon$.

The proofs of Theorem 1.1 and Corollary 1.4 are given in Sect. 3 of this paper.

\section{Periodic Points and Local Minima of Length Functions}

In this section we assume that $Q$ satisfies the assumptions at the beginning of Sect. 1 . Denote by $K_{i}$ the convex hull of $\Gamma_{i}$ in $\mathbb{R}^{d}$. Then $K_{i}$ is a compact convex subset of $\mathbb{R}^{d}$ and $\Gamma_{i} \subset \partial K_{i}$ by the main assumption (cf. Sect. 1). It may occur that $K_{i}$ and $K_{j}$ have common interior points for some $i \neq j$, but this will not interfere with our considerations.

Fix an $\alpha=\left(i_{1}, \ldots, i_{k}\right) \in \mathscr{A}_{k}$. For convenience we set $q_{k+1}=q_{1}$ and $q_{0}=q_{k}$. Consider the length function

$$
F=F_{\alpha}: K_{\alpha}=K_{i_{1}} \times \cdots \times K_{i_{k}} \rightarrow \mathbb{R}
$$

defined by

$$
F\left(q_{1}, \ldots, q_{k}\right)=\sum_{j=1}^{k}\left\|q_{j}-q_{j+1}\right\| .
$$

Clearly, if $(q, v)$ is a periodic point of type $\alpha$ for $T$, then for $q_{j}=\pi^{\circ} T^{j-1}(q, v)$ we have that $F\left(q, \ldots, q_{k}\right)$ is the length of the corresponding periodic billiard trajectory.

Set $\Gamma_{\alpha}=\Gamma_{i_{1}} \times \cdots \times \Gamma_{i_{k}} \subset K_{\alpha}$ (this is not the boundary of $K_{\alpha}$ in $\left.\left(\mathbb{R}^{d}\right)^{k}\right)$. It is well-known that if the restriction $F_{\mid \Gamma_{\alpha}}$ of $F$ to $\Gamma_{\alpha}$ has a local minimum at some point $\tilde{q}=\left(q_{1}, \ldots, q_{k}\right) \in \Gamma_{\alpha}$ and if for every $j=1, \ldots, k$ the open segment $\left(q_{j}, q_{j+1}\right)$ is contained in the interior of $Q$, then $q_{1}, \ldots, q_{k}$ are the consecutive reflection points of a periodic billiard trajectory in $Q$. Our aim in this section is to prove the converse.

Lemma 2.1. Let $(q, v)$ be a periodic point of type $\alpha$ for $T$ and let $q_{j}=\pi \circ T^{j-1}(q, v)$, $j=1, \ldots, k$. Then $F$ has a local minimum at $\tilde{q}=\left(q_{1}, \ldots, q_{k}\right)$ as a function on $K_{\alpha}$.

Proof. Clearly, $F$ is smooth in a neighbourhood of $\tilde{q}$. Since the case $k=2$ is clear, we will assume $k \geqq 3$.

Every $q_{j}$ is a regular point of $\Gamma$, therefore there is a $C^{2}$-smooth cart

$$
\varphi_{j}: \mathbb{R}^{d-1} \rightarrow U_{j} \subset \Gamma_{i_{j}}
$$

such that $\varphi_{j}(0)=q_{j}$. Then $\left.\left\{\partial \varphi_{j} / \partial u_{j}^{(n)}\right)(0)\right\}_{n=1}^{d-1}$ is a basis in the tangent space $T_{q_{j}} \Gamma$ to $\Gamma$ at $q_{j}$. Hence $u_{j}=\left(u_{j}^{(1)}, \ldots, u_{j}^{(d-1)}\right)$ belongs to $\mathbb{R}^{d-1}$. Consider the function

$$
G:\left(\mathbb{R}^{d-1}\right)^{k} \rightarrow \mathbb{R}
$$

defined by

$$
G\left(u_{1}, \ldots, u_{k}\right)=F\left(\varphi_{1}\left(u_{1}\right), \ldots, \varphi_{k}\left(u_{k}\right)\right) .
$$

First, we are going to prove that $G$ has a local minimum at 0 . This would imply that $F_{\mid \Gamma_{\alpha}}$ has a local minimum at $\tilde{q}$.

Let $\varphi_{j}\left(u_{j}\right) \stackrel{\alpha}{=}\left(\varphi_{j}^{(1)}\left(u_{j}\right), \ldots, \varphi_{j}^{(d)}\left(u_{j}\right)\right)$, and let $u=\left(u_{1}, \ldots, u_{k}\right) \in\left(\mathbb{R}^{d-1}\right)^{k}$. In what follows we will use the following notation: $I_{j}=\{j-1, j+1\}$,

$$
a_{j i}=1 /\left\|q_{j}-q_{i}\right\|, v_{j i}=\left(q_{j}-q_{i}\right) /\left\|q_{j}-q_{i}\right\| \quad\left(i \in I_{j}\right) .
$$


Clearly, $a_{j i}>0$ and $v_{j i} \in S^{d-1}$. Moreover, $a_{i j}=a_{j i}$ and $v_{i j}=-v_{j i}$.

For all $j=1, \ldots, k, n=1, \ldots, d-1$ and $u$ sufficiently close to 0 we have

$$
\frac{\partial G}{\partial u_{j}^{(n)}}(u)=\sum_{i \in I_{j}}\left\langle\frac{\varphi_{j}\left(u_{j}\right)-\varphi_{i}\left(u_{i}\right)}{\left\|\varphi_{j}\left(u_{j}\right)-\varphi_{i}\left(u_{i}\right)\right\|}, \frac{\partial \varphi_{j}}{\partial u_{j}^{(n)}}\left(u_{j}\right)\right\rangle .
$$

Since $v_{j j-1}+v_{j j+1}$ is collinear with $N\left(q_{j}\right)$, one gets

$$
\frac{\partial G}{\partial u_{j}^{(n)}}(0)=\left\langle v_{j j-1}+v_{j j+1}, \frac{\partial \varphi_{j}}{\partial u_{j}^{(n)}}(0)\right\rangle=0 .
$$

Therefore 0 is a critical point of $G$.

Next, we will show that the second fundamental form of $G$ at 0 is non-negative definite. First, we have to compute $\left(\partial^{2} G / \partial u_{j}^{(n)} \partial u_{i}^{(m)}\right)(0)$ for all $j, i=1, \ldots, k$ and $n, m=1, \ldots, d-1$. Given $j$ there are three possibilities for $i$.

Case 1. $i \notin I_{j} \cup\{j\}$. Then $\left(\partial^{2} G / \partial u_{j}^{(n)} \partial u_{i}^{(m)}\right)(0)=0$.

Case 2. $i \in I_{j}$. Now (3) implies

$$
\begin{aligned}
\frac{\partial^{2} G}{\partial u_{j}^{(n)} \partial u_{i}^{(m)}}(0)= & -a_{j i}\left\langle\frac{\partial \varphi_{j}}{\partial u_{j}^{(n)}}(0), \frac{\partial \varphi_{i}}{\partial u_{i}^{(m)}}(0)\right\rangle \\
& +a_{j i}\left\langle\frac{\partial \varphi_{j}}{\partial u_{j}^{(n)}}(0), v_{j i}\right\rangle\left\langle\frac{\partial \varphi_{i}}{\partial u_{i}^{(m)}}(0), v_{j i}\right\rangle .
\end{aligned}
$$

Case 3. $i=j$. Then

$$
\begin{aligned}
\frac{\partial^{2} G}{\partial u_{j}^{(n)} \partial u_{j}^{(m)}}(0)= & \sum_{i \in I_{j}}\left\langle v_{j i}, \frac{\partial^{2} \varphi_{j}}{\partial u_{j}^{(n)} \partial u_{j}^{(m)}}(0)\right\rangle+\sum_{i \in I_{j}} a_{j i}\left\langle\frac{\partial \varphi_{j}}{\partial u_{j}^{(n)}}(0), \frac{\partial \varphi_{j}}{\partial u_{j}^{(m)}}(0)\right\rangle \\
& -\sum_{i \in I_{j}} a_{j i}\left\langle\frac{\partial \varphi_{j}}{\partial u_{j}^{(n)}}(0), v_{j i}\right\rangle\left\langle\frac{\partial \varphi_{j}}{\partial u_{j}^{(m)}}(0), v_{j i}\right\rangle .
\end{aligned}
$$

Fix an arbitrary vector $\xi=\left(\xi_{j}^{(n)}\right)_{1 \leqq J \leqq k, 1 \leqq n \leqq d-1}$ in $\left(\mathbb{R}^{d-1}\right)^{k}$. We have to show that

$$
\sigma=\sum_{j, i=1}^{k} \sum_{n, m=1}^{d-1} \frac{\partial^{2} G}{\partial u_{j}^{(n)} \partial u_{i}^{(m)}}(0) \xi_{j}^{(n)} \xi_{i}^{(m)} \geqq 0 .
$$

Set $z_{j}=\sum_{n=1}^{d-1} \xi_{j}^{(n)}\left(\partial \varphi_{j} / \partial u_{j}^{(n)}\right)(0)$, where $\xi_{j}=\left(\xi_{j}^{(1)}, \ldots, \xi_{j}^{(d-1)}\right)$. Note that for $N_{j}=N\left(q_{j}\right)$ we have $v_{j j-1}+v_{j j+1}=-\lambda_{j} N_{j}$ for some $\lambda_{j}>0$.

Since $U_{j}=\varphi_{j}\left(\mathbb{R}^{d-1}\right) \subset \Gamma$ is convex at $q_{j}$, the choice of the normal vector $N_{j}$ shows that the second fundamental form $B_{j}$ of $U_{j}$ at $q_{j}$ is non-positive definite. That is

$$
B_{j}\left(\xi_{j}, \xi_{j}\right)=\sum_{n, m=1}^{d-1}\left\langle N_{j}, \frac{\partial^{2} \varphi_{j}}{\partial u_{j}^{(n)} \partial u_{j}^{(m)}}(0)\right\rangle \xi_{j}^{(n)} \xi_{j}^{(m)} \leqq 0
$$

for every $\xi_{j} \in \mathbb{R}^{d-1}$.

According to the above formulas for the second derivatives of $G$ at 0 we find: 


$$
\begin{aligned}
\sigma= & \sum_{j=1}^{k} \sum_{n, m=1}^{d-1} \frac{\partial^{2} G}{\partial u_{j}^{(n)} \partial u_{j}^{(m)}}(0) \xi_{j}^{(n)} \xi_{j}^{(m)} \\
& +\sum_{j=1}^{k} \sum_{i \in I_{j}} \sum_{n, m=1}^{d-1} \frac{\partial^{2} G}{\partial u_{j}^{(n)} \partial u_{i}^{(m)}}(0) \xi_{j}^{(n)} \xi_{i}^{(m)} \\
= & -\sum_{j=1}^{k} \lambda_{j} \sum_{n, m=1}^{d-1}\left\langle N_{j}, \frac{\partial^{2} \varphi_{j}}{\partial u_{j}^{(n)} \partial u_{j}^{(m)}}(0)\right\rangle \xi_{j}^{(n)} \xi_{j}^{(m)} \\
& +\sum_{j=1}^{k} \sum_{i \in I_{j}} \sum_{n, m=1}^{d-1} a_{j i}\left\langle\frac{\partial \varphi_{j}}{\partial u_{j}^{(n)}}(0), \frac{\partial \varphi_{j}}{\partial u_{j}^{(m)}}(0)\right\rangle \xi_{j}^{(n)} \xi_{j}^{(m)} \\
& \left.-\sum_{j=1}^{k} \sum_{i \in I_{j}} \sum_{n, m=1}^{d-1} a_{j i}\left\langle\frac{\partial \varphi_{j}}{\partial u_{j}^{(n)}}(0), v_{j i}\right\rangle\left\langle\frac{\partial \varphi_{j}}{\partial u_{j}^{(m)}}(0), v_{j i}\right\rangle \xi_{j}^{(n)} \xi_{j}^{(m)}\right] \\
& +\left[-\sum_{j=1}^{k} \sum_{i \in I_{j}} \sum_{n, m=1}^{d-1} a_{j i}\left\langle\frac{\partial \varphi_{j}}{\partial u_{j}^{(n)}}(0), \frac{\partial \varphi_{i}}{\partial u_{i}^{(m)}}(0)\right\rangle \xi_{j}^{(n)} \xi_{i}^{(m)}\right. \\
& \left.+\sum_{j=1}^{k} \sum_{i \in I_{j}} \sum_{n, m=1}^{d-1} a_{j i}\left\langle\frac{\partial \varphi_{j}}{\partial u_{j}^{(n)}}(0), v_{j i}\right\rangle\left\langle\frac{\partial \varphi_{i}}{\partial u_{i}^{(m)}}(0), v_{j i}\right\rangle \xi_{j}^{(n)} \xi_{i}^{(m)}\right] \\
= & -\sum_{j=1}^{k} \lambda_{j} B_{j}\left(\xi_{j}, \xi_{j}\right)+\sum_{j=1}^{k} \sum_{i \in I_{j}} a_{j i}\left\langle z_{j}, z_{j}\right\rangle-\sum_{j=1}^{k} \sum_{i \in I_{j}} a_{j i}\left\langle z_{j}, v_{j i}\right\rangle^{2} \\
& -\sum_{j=1}^{k} \sum_{i \in I_{j}} a_{j i}\left\langle z_{j}, z_{i}\right\rangle+\sum_{j=1}^{k} \sum_{i \in I_{j}} a_{j i}\left\langle z_{j}, v_{j i}\right\rangle\left\langle z_{i}, v_{j i}\right\rangle .
\end{aligned}
$$

Since $i \in I_{j}$ is equivalent to $j \in I_{i}$, according to $a_{j i}=a_{i j}$ and $v_{j i}=-v_{i j}$, one can rewrite the last expression for $\sigma$ as follows:

$$
\begin{aligned}
\sigma= & -\sum_{j=1}^{k} \lambda_{j} B_{j}\left(\xi_{j}, \xi_{j}\right)+\sum_{j=1}^{k} a_{j j+1}\left[\left\|z_{j}\right\|^{2}-\left\langle z_{j}, v_{j j+1}\right\rangle^{2}\right. \\
& -\left\langle z_{j}, z_{j+1}\right\rangle+\left\langle z_{j}, v_{j j+1}\right\rangle\left\langle z_{j+1}, v_{j j+1}\right\rangle+\left\|z_{j+1}\right\|^{2} \\
& \left.-\left\langle z_{j+1}, v_{j+1 j}\right\rangle^{2}-\left\langle z_{j+1}, z_{j}\right\rangle+\left\langle z_{j+1}, v_{j+1 j}\right\rangle\left\langle z_{j}, v_{j+1 j}\right\rangle\right] \\
= & -\sum_{j=1}^{k} \lambda_{j} B_{j}\left(\xi_{j}, \xi_{j}\right)+\sum_{j=1}^{k} a_{j j+1}\left[\left\|z_{j}-z_{j+1}\right\|^{2}-\left\langle z_{j}-z_{j+1}, v_{j j+1}\right\rangle^{2}\right] .
\end{aligned}
$$

By definition $\left\|v_{j j+1}\right\|=1$, therefore $\left\langle z_{j}-z_{j+1}, v_{j j+1}\right\rangle^{2} \leqq\left\|z_{j}-z_{j+1}\right\|^{2}$, which yields $\sigma \geqq 0$.

In this way we have shown that $G$ has a local minimum at 0 , thus the restriction of $F$ to $\Gamma_{\alpha}$ has a local minimum at $\tilde{q}$. Then there exist neighbourhoods $V_{j}$ of $q_{j}$ in $K_{i_{j}}$ such that $F(\tilde{q}) \leqq F(\tilde{p})$ for every $\tilde{p} \in V \cap \Gamma_{\alpha}$, where $V=V_{1} \times \cdots \times V_{k}$. Since $T^{j-1}(q, v)$ are admissible points for all $j \geqq 1$, we may choose the neighbourhoods $V_{j}$ in such a way that for every $\tilde{p} \in V$ and every $j=1, \ldots, k$ the segment $\left[p_{j}, p_{j+1}\right]$ intersects $\Gamma_{i_{j}}$ and $\Gamma_{i_{j+1}}$ at points belonging to $V_{j}$ and $V_{j+1}$, respectively. Indeed, if $q_{j}$ is a tangent reflection point, we may define $V_{j}$ by

$$
V_{j}=\left\{p_{j} \in K_{i_{j}}:\left\langle p_{j}-q_{j}, N\left(q_{j}\right)\right\rangle>-\varepsilon_{j}\right\}
$$


for some $\varepsilon_{j}>0$. If $q_{j}$ is a proper reflection point, we take an open ball $D_{j}$ with center $q_{j}$ and a sufficiently small radius $\varepsilon_{j}>0$ and set $V_{j}=K_{i_{j}} \cap D_{j}$.

Consider an arbitrary $\tilde{p}=\left(p_{1}, \ldots, p_{k}\right) \in V$. Denote by $p_{1}^{\prime}$ the intersection point of $\Gamma_{i_{1}}$ and the segment $\left[p_{1}, p_{2}\right]$. Then $p_{1}^{\prime} \in V_{1}$, and it follows by the triangle inequality that

$$
F\left(p_{1}, p_{2}, \ldots, p_{k}\right) \geqq F\left(p_{1}^{\prime}, p_{2}, \ldots, p_{k}\right) .
$$

Next, denoting by $p_{2}^{\prime}$ the intersection point of $\Gamma_{i_{2}}$ and the segment $\left[p_{1}, p_{2}\right]$ we obtain

$$
F\left(p_{1}^{\prime}, p_{2}, p_{3}, \ldots, p_{k}\right) \geqq F\left(p_{1}^{\prime}, p_{2}^{\prime}, p_{3}, \ldots, p_{k}\right),
$$

and so on. Thus we find for each $j, p_{j}^{\prime} \in \Gamma_{i_{j}} \cap V_{j}$ such that $F(\tilde{p}) \geqq F\left(\tilde{p}^{\prime}\right)$, where $\tilde{p}^{\prime}=\left(p_{1}^{\prime}, \ldots, p_{k}^{\prime}\right) \in \Gamma_{\alpha} \cap V$. It follows from above that $F\left(\tilde{p}^{\prime}\right) \geqq F(\tilde{q})$, therefore $F(\tilde{p}) \geqq F(\tilde{q})$. This proves the assertion.

Remark. If $\Gamma_{i}$ is strictly convex at $q_{j}$ for every $j$, then clearly $F$ has a strict local minimum at $\tilde{q}$.

\section{Proofs of the Main Results}

Let $Q$ be as at the beginning of Sect. 1 and let $\alpha \in \mathscr{A}_{k}$ be given. In what follows we will use the function (1) defined by (2). Note that $F$ is convex, that is

$$
F(t \tilde{q}+(1-t) \tilde{p}) \leqq t F(\tilde{q})+(1-t) F(\tilde{p})
$$

for all $\tilde{q}, \tilde{p} \in K_{\alpha}$ and $t \in[0,1]$.

Proof of Theorem 1.1. Assume there exist two different periodic points $(q, v)$ and $(p, w)$ of type $\alpha$ for $T$. Set $\tilde{q}=\left(q_{1}, \ldots, q_{k}\right)$ and $\tilde{p}=\left(p_{1}, \ldots, p_{k}\right)$. Then $\tilde{q}, \tilde{p} \in K_{\alpha}$ and by Lemma $2.1 F$ has local minima at $\tilde{q}$ and $\tilde{p}$. For $t \in[0,1]$ set $q_{j}^{(t)}=t q_{j}+(1-t) p_{j}$ and $\tilde{q}^{(t)}=\left(q_{1}^{(t)}, \ldots, q_{k}^{(t)}\right)$. Clearly, $\tilde{q}^{(t)}=t \tilde{q}+(1-t) \tilde{p} \in K_{\alpha}$.

We will show that $F(\tilde{q})=F(\tilde{p})$. Assume $F(\tilde{q})>F(\tilde{p})$. Then for every $t \in(0,1)$ we have

$$
F\left(\tilde{q}^{(t)}\right)=F(t \tilde{q}+(1-t) \tilde{p}) \leqq t F(\tilde{q})+(1-t) F(\tilde{p})<F(\tilde{q}) .
$$

Since $\tilde{q}^{(t)} \rightarrow \tilde{q}$ as $t \rightarrow 1$, we get a contradiction with the fact that $F$ has a local minimum at $\tilde{q}$. Thus $F(\tilde{q}) \leqq F(\tilde{p})$. Similarly one gets $F(\tilde{p}) \leqq F(\tilde{q})$, therefore $F(\tilde{q})=F(\tilde{p})$. Moreover, by $F\left(\tilde{q}^{(t)}\right)=F(t \tilde{q}+(1-t) \tilde{p}) \leqq F(\tilde{q})=F(\tilde{p})$ we find that $F\left(\tilde{q}^{(t)}\right)=F(\tilde{q})=F(\tilde{p})$ for all $t \in(0,1)$ sufficiently close to 0 or 1 . It then follows that $F\left(\tilde{q}^{(t)}\right)=F(\tilde{q})=F(\tilde{p})$ for all $t \in[0,1]$. Note that for $t \in(0,1)$ the equality

$$
\left\|[t q+(1-t) p]-\left[t q^{\prime}+(1-t) p^{\prime}\right]\right\|=t\left\|q-q^{\prime}\right\|+(1-t)\left\|p-p^{\prime}\right\|
$$

holds if and only if the segments $\left[q, q^{\prime}\right]$ and $\left[p, p^{\prime}\right]$ are parallel (we assume $q \neq q^{\prime}$ and $\left.p \neq p^{\prime}\right)$. Then it follows from above that the segments $\left[q_{j}, q_{j+1}\right]$ and $\left[p_{j}, p_{j+1}\right]$ are parallel for each $j=1,2, \ldots$. In particular, $v=w$.

Take neighbourhoods $V_{j}$ of $q_{j}$ in $K_{i_{j}}$ as at the end of Sect. 2. There exists $t_{0} \in(0,1)$ such that $q_{j}^{(t)} \in V_{j}$ for all $t \in\left(t_{0}, 1\right]$. Set $V=V_{1} \times \cdots \times V_{k}$. Clearly, $F$ has a minimum at $\tilde{q}^{(t)}$ in $V$ for every $t \in\left(t_{0}, 1\right]$. Let $q_{j}$ be a proper reflection point for some $j \leqq k$, and suppose $q_{j}^{(t)} \notin \Gamma_{i_{j}}$ for some $t \in\left(t_{0}, 1\right)$. Set $\tilde{r}=\left(q_{1}^{(t)}, \ldots, q_{j-1}^{(t)}, q_{j}^{\prime}, q_{j+1}^{(t)}, \ldots\right.$, 
$\left.q_{k}^{(t)}\right)$, where $q_{j}^{\prime}$ is the point of intersection of $\Gamma_{i_{j}}$ and the segment $\left[q_{j}^{(t)}, q_{j+1}^{(t)}\right]$. Since $q_{j}$ is a proper reflection point, if $t_{0}$ is sufficiently close to 1 , then the segments $\left[q_{j-1}^{(t)}, q_{j}^{(t)}\right]$ and $\left[q_{j}^{(t)}, q_{j+1}^{(t)}\right]$ would be not collinear, so

$$
\left\|q_{j-1}^{(t)}-q_{j}^{(t)}\right\|+\left\|q_{j}^{(t)}-q_{j+1}^{(t)}\right\|>\left\|q_{j-1}^{(t)}-q_{j}^{\prime}\right\|+\left\|q_{j}^{\prime}-q_{j+1}^{(t)}\right\|,
$$

and therefore $F\left(\tilde{q}^{(t)}\right)>F(\tilde{r})$ in contradiction with the minimality of $F\left(\tilde{q}^{(t)}\right)$. Hence $q_{j}^{(t)} \in \Gamma_{i_{j}}$ for all $t \in\left(t_{0}, 1\right]$ providing $t_{0}$ is sufficiently close to 1 .

Finally, if all $q_{1}, \ldots, q_{k}$ are proper reflection points, then it follows from above that for every $t \in(0,1)$ sufficiently close to 1 the points $(t q+(1-t) p, v)$ are periodic points of type $\alpha$ for $T$ which generate periodic billiard trajectories in $Q$ of length $F(\tilde{q})=F(\tilde{p})$ and parallel corresponding segments.

Proof of Corollary 1.4. Let $i \neq j$ be such that $\Gamma_{i} \cap \Gamma_{j} \neq \varnothing$ and let $q \in \Gamma_{i} \cap \Gamma_{j}$. Denote by $\omega_{i j}(q)$ the minimal angle between two different tangents to $\Gamma_{i}$ and $\Gamma_{j}$ at $q$. Put

$$
\omega=\min \left\{\omega_{i j}(q): i \neq j, q \in \Gamma_{i} \cap \Gamma_{j}\right\}
$$

if the set on the right-hand side is non-empty, and $\omega=\pi$ otherwise. For $n=[\pi / 2 \omega]+1$ a simple geometrical argument shows that if $\gamma(x), x \in M_{0}$, is a billiard semi-trajectory in $Q$ and if $\Gamma_{i} \cap \Gamma_{j} \neq \varnothing$, then there are no more than $2 n$ consecutive reflection points of $\gamma(x)$ belonging to $\Gamma_{i} \cup \Gamma_{j}$.

Further, divide each $\Gamma_{i}$ which has endpoints into two curves $\Gamma_{i}^{\prime}$ and $\Gamma_{i}^{\prime \prime}$ by an arbitrary point $q_{i} \in \Gamma_{i}\left(\Gamma_{i}=\Gamma_{i}^{\prime} \cup \Gamma_{i}^{\prime \prime}\right.$ and $\Gamma_{i}^{\prime} \cap \Gamma_{i}^{\prime \prime}=\left\{q_{i}\right\}$ if $\Gamma_{i}$ has two different endpoints, $\Gamma_{i}^{\prime} \cap \Gamma_{i}^{\prime \prime}=\left\{q_{i}\right\} \cup \partial \Gamma_{i}$ otherwise). If $\partial \Gamma_{i}=\varnothing$, i.e. $\Gamma_{i}$ has no endpoints, set $\Gamma_{i}^{\prime}=\Gamma_{i}^{\prime \prime}=\Gamma_{i}$. Define the numbers

$$
\begin{aligned}
m_{i}^{\prime} & =\min \left\{\operatorname{dist}\left(\Gamma_{i}^{\prime}, \Gamma_{j}\right): \Gamma_{j} \cap \Gamma_{i}^{\prime}=\varnothing\right\}, \\
m_{i}^{\prime \prime} & =\min \left\{\operatorname{dist}\left(\Gamma_{i}^{\prime \prime}, \Gamma_{j}\right): \Gamma_{j} \cap \Gamma_{i}^{\prime \prime}=\varnothing\right\}, \\
m & =\min \left\{m_{1}^{\prime}, \ldots, m_{s}^{\prime}, m_{1}^{\prime \prime}, \ldots, m_{s}^{\prime \prime}\right\} .
\end{aligned}
$$

Clearly, $m>0$. Moreover, it follows from above that if $p_{k}, p_{k+1}, \ldots, p_{k+2 n}$ are consecutive reflection points of a billiard semi-trajectory $\gamma(x)$ in $Q, x \in M_{0}$, then at least one of the segments $\left[p_{j}, p_{j+1}\right], j=1, \ldots, k+2 n-1$, has a length not less than $m$.

Take an arbitrary $t>0$, and let $\gamma=\gamma(x), x \in M_{0}$, be an arbitrary periodic billiard trajectory in $Q$ with length $l_{\gamma} \leqq t$. If $k$ is the number of reflections of $\gamma$, then

$$
l_{\gamma} \geqq m[k /(2 n+1)] \geqq m(k-2 n) /(2 n+1),
$$

so $k \leqq(2 n+1) t / m+2 n$. Therefore for $i=[(2 n+1) t / m]$, according to Corollary 1.3 , we find

$$
\widetilde{P}_{t} \leqq \sum_{j=2}^{i+2 n} P_{j}<\sum_{j=2}^{i+2 n}(s-1)^{j}<(s-1)^{i+2 n} \leqq(s-1)^{c t+b},
$$

where $c=(2 n+1) / m$ and $b=2 n+1$. This proves the assertion.

Acknowledgements. I am grateful to Ya. Sinai and N. Chernov for their remarks and suggestions, to A. Kramli who read the whole text, and to V. Petkov for many useful discussions on the subject of this paper. 


\section{References}

1. Bowen, R.: Topological entropy and Axiom A. Proc. Symp. Pure Math. vol. 14, pp.23-41, Providence R.I.: A.M.S. 1970

2. Bunimovich, L. A.: Hyperbolic systems with singularities. In: Dynamical Systems II (Encyclopedia of Mathematical Sciences, vol. 2), Sinai Ya. (ed.). Berlin, Heidelberg, New York: Springer 1987

3. Bunimovich, L. A., Sinai, Ya. G., Chernov, N. I.: Markov partitions for two-dimensional dispersed billiards and near systems (Russian). Uspehi Mat. Nauk (to appear)

4. Cornfeld, I. P., Fomin, S. V., Sinai, Ya. G.: Ergodic theory. Berlin, Heidelberg, New York: Springer 1982

5. Ikawa, M.: Decay of solutions of the wave equation in the exterior of several convex bodies. Ann. Inst. Fourier 38, 113-146 (1988)

6. Ikawa, M.: On the poles of the scattering matrix for several convex bodies. Preprint 1987

7. Katok, A.: Lyapunov exponents, entropy and periodic orbits for diffeomorphisms. Publ. Math. I.H.E.S. 51, 137-173 (1980)

8. Katok, A.: Entropy and closed geodesics. Ergod. Theory Dynam. Sys. 2, 339-367 (1982)

9. Katok, A.: The growth rate of the number of singular and periodic orbits for a polygonal billiard. Commun. Math. Phys. 111, 151-160 (1987)

10. Katok, A., Strelcyn, J.-M.: In collaboration with Ledrappier, F., Przytycki, F.: Smooth maps with singularities: Invariant manifolds, entropy and billiards. Lecture Notes in Mathematics, vol. 122. Berlin, Heidelberg, New York: Springer 1986

11. Kramli, A., Simanyi, N., Szasz, D.: Three billiard balls on the $v$-dimensional torus is a $K$-flow. Preprint No. 38/1988, Math. Institute of the Hungarian Academy of Sciences

12. Margulis, G.: Applications of ergodic theory to the investigation of manifolds of negative curvature. Funct. Anal. Appl. 3, 335-336 (1969)

13. Pesin, Ya. B., Sinai, Ya. G.: Hyperbolicity and Stochasticity of Dynamical Systems pp. 53-116. London: Gordon and Breach Press 1981

14. Petkov, V., Stojanov, L.: On the number of periodic reflecting rays in generic domains. Ergod. Theory Dynam. Sys. 8, 81-91 (1988)

15. Sinai, Ya. G.: Dynamical systems with elastic reflections. Ergodic properties of dispersing billiards. Russ. Math. Surveys 25, 137-189 (1970)

16. Sinai, Ya. G.: Development of Krylov's ideas. An addendum to the book: Krylov, N. S.: Works on the foundation of statistical physics. pp. 239-281, Princeton, NJ: Princeton University Press 1979

17. Sinai, Ya. G., Chernov, N.I.: Ergodic properties of some systems of two-dimensional discs and three-dimensional spheres (Russian). Uspehi Mat. Nauk 42, 153-174 (1987)

18. Wojtkowski, M.: Principles for the design of billiards with nonvanishing Lyapunov exponents. Commun. Math. Phys. 105, 391-414 (1986)

\section{Communicated by Ya. G. Sinai}

Received August 22, 1988 
